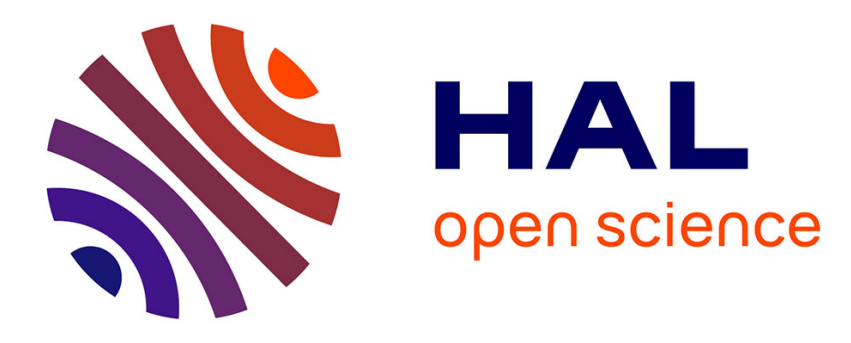

\title{
Les limites actuelles de l'apprentissage collaboratif en ligne
}

\author{
Michel Arnaud
}

\section{To cite this version:}

Michel Arnaud. Les limites actuelles de l'apprentissage collaboratif en ligne. STICEF (Sciences et Technologies de l'Information et de la Communication pour l'Éducation et la Formation), 2003, 10, 7 p. hal-00696421

\section{HAL Id: hal-00696421 https://hal.science/hal-00696421}

Submitted on 11 May 2012

HAL is a multi-disciplinary open access archive for the deposit and dissemination of scientific research documents, whether they are published or not. The documents may come from teaching and research institutions in France or abroad, or from public or private research centers.
L'archive ouverte pluridisciplinaire HAL, est destinée au dépôt et à la diffusion de documents scientifiques de niveau recherche, publiés ou non, émanant des établissements d'enseignement et de recherche français ou étrangers, des laboratoires publics ou privés. 


\title{
Les limites actuelles de l'apprentissage collaboratif en ligne
}

\author{
Michel Arnaud [CRIS SERIES]
}

RÉSUMÉ : L'examen d'une pratique collaborative à distance au travers des trois premières promotions du DESS UTICEF de Strasbourg 1, montre un éventail de comportements de la part des étudiants allant de l'adhésion à la collaboration, à une préférence pour la coopération et enfin à la persistance du travail en solitaire. Ces attitudes s'insèrent dans trois profils d'apprenants : accrocheurs, sérieux, indépendants. Notre analyse nous incite à rester prudents quand il est envisagé d'appliquer le modèle collaboratif à des cohortes nombreuses en utilisant des outils informatiques permettant de démultiplier l'action des tuteurs.

\section{Introduction}

L'apprentissage collaboratif en ligne est un dispositif encore nouveau dans les universités françaises et francophones du Sud. L'observation des trois premières promotions du DESS UTICEF (Utilisation des Technologies de l'Information et de la Communication pour l'Enseignement et la Formation) de Strasbourg 1 a permis de mesurer les réactions des étudiants à l'usage du dispositif d'apprentissage collaboratif proposé, à savoir l'utilisation de salons virtuels de travaux dirigés. Si une majorité d'étudiants sait en tirer profit, une minorité reste réfractaire. Les réactions négatives relevées dans cette analyse peuvent s'expliquer par la persistance de pratiques caractéristiques de l'enseignement présentiel encore prégnantes dans les usages et comportements qui s'installent à distance. Les étudiants du DESS UTICEF sont recrutés dans le cadre de la formation continue. Ce sont des enseignants du secondaire et surtout du supérieur, issus de disciplines variées, en provenance de toutes les zones francophones du Sud, ainsi que des professionnels des secteurs public et privé du Nord et du Sud. Ils s'inscrivent pour pouvoir développer l'usage des TIC dans leur organisation, mettre en ligne des cours dans leur spécialité par exemple, projet personnel qu'ils doivent réaliser en fin de session sous forme d'un prototype.

Il convient de replacer la présente analyse dans la perspective d'une évolution possible dans le temps, qui ferait que l'apprentissage collaboratif en ligne serait mieux accepté par davantage d'étudiants plus familiers avec ce type de pratique, à l'image des formations dispensées par l'Open University au Royaume Uni, la Téluq au Canada, l'Université de Mons en Belgique, l'Université de Genève. Toutefois, de nombreux échecs se sont produits de par le monde et continuent à être constatés dans le domaine de l'apprentissage en ligne, que ce soit au niveau des outils, des dispositifs et des usages proposés. La première phase du "e-learning" dans les entreprises est retombée avec la bulle Internet en 2001. Le retour sur investissement ne s'est pas produit du fait des coûts de mise en ligne des contenus et de l'usage de plateformes propriétaires d'enseignement en ligne, le taux de réussite étant par ailleurs trop faible par rapport au taux d'abandon.

Les obstacles à la mise en ligne de formations universitaires sont d'ordre technologique du fait de la complexité des réseaux et d'ordre financier avec la nécessité d'une masse critique d'inscrits pour amortir les coûts de production, de diffusion et de suivi pédagogique. Par ailleurs, les concepteurs, tuteurs et étudiants doivent acquérir des compétences spécifiques pour pouvoir tirer profit des dispositifs proposés. Les concepteurs doivent être formés pour que les contenus soient présentés de manière propice à la collaboration, de même que les tuteurs afin qu'ils s'adaptent au suivi des échanges collaboratifs. Comme le remarque Peeters et al. [PeetersA199], les étudiants doivent développer des compétences sociales.

L'apprentissage en ligne n'a pas de modèle de référence parce qu'il n'y a pas de recherche qui en ait démontré l'efficacité, du fait de la difficulté d'isoler les variables entrant en ligne de compte. Aucune certitude n'existe du point de vue de la psychologie cognitive sur le type exact d'interactions entre l'étudiant, l'écran, le groupe virtuel et le contenu en ligne : trop de variables sont concernées, comme le font remarquer Tricot et al. [TricotA198]. Les variables après coup permettent de mesurer le degré de satisfaction des étudiants, la compréhension et mémorisation des contenus traités, le contexte de l'activité d'apprentissage. Mais comment définir l'activité mentale d'un utilisateur d'hypermédia ? Comment mesurer l'apport de la collaboration dans le processus d'écriture collective du document d'équipe ? Comment évaluer l'efficacité de tel ou tel outil dans le processus de collaboration, lorsque la mise en situation introduit d'autres variables liées au comportement spécifique de chaque étudiant ? Comme le note Cerratto [Cerrato99], la médiatisation de la relation humaine s'ajoute à celle des contenus. Pour être efficaces, les environnements d'apprentissage collaboratif à distance avec usage d'outils d'échanges synchrones et asynchrones demandent une forte médiation humaine. 


\section{Les profils selon les comportements et usages des outils}

Le DESS UTICEF est un diplôme dispensé pratiquement entièrement à distance. Il comporte seulement quatre jours de regroupement en début de session pour que les étudiants se familiarisent avec la plateforme Acolad, mise au point par le service commun de l'université Louis Pasteur (ULPMultimédia), et toutes les fonctionnalités qu'elle propose : accéder à son propre espace de travail (bureau virtuel), aux salons de chats communs à toute la promotion, aux cours auxquels les étudiants sont inscrits et aux séminaires virtuels par groupe de 10-12 étudiants et par équipes de 2-4 étudiants, utiliser le service permettant de communiquer en privé avec quiconque se trouvant sur la plateforme.

Des contraintes temporelles sont données tout au long de l'année de telle sorte que le travail de groupe soit rendu à un moment donné dans le cadre de chaque module d'enseignement. La planification du travail en découle au sein de l'équipe, ce qui est censé éviter les dispersions et déperditions d'énergie. La présence aux réunions synchrones est obligatoire ; il y en a trois à quatre par module, réparties sous forme de plages de plusieurs heures de chat. La question de la disponibilité des participants se pose fréquemment du fait de leurs occupations professionnelles, des jours fériés et activités privées et de la compatibilité des plages horaires. Il se produit une mise en perspective du travail réalisé en solo et en groupe au cours de ces réunions qui servent à valider et relancer le processus de production du document final si le tuteur le juge nécessaire. Elles servent aussi à mesurer le degré d'implication des étudiants concernés.

$\mathrm{Au}$ fil des séminaires virtuels suivis par les trois premières promotions, soit 94 étudiants, l'observation des usages des réunions synchrones et d'une manière générale, l'étude des comportements, ont permis de dégager trois types de profils, mis à part ceux qui ont des problèmes de connexion. La distribution des variables (connexion facile, présence aux séminaires, motivation pour le travail d'équipe), permet d'opérer les premières différenciations.

La variable de la connexion facile a été conservée dans le tableau permettant de définir les profils d'étudiants, car elle représente en général l'excuse la plus utilisée pour justifier l'absence aux réunions virtuelles synchrones et la non participation aux échanges virtuels asynchrones. Il existe par ailleurs des difficultés considérables d'accès aux réseaux dans les pays du Sud. Le fait de pouvoir facilement se connecter est encore le privilège des personnes habitant dans les capitales du Sud. Par conséquent, 9 étudiants trop excentrés ont été exclus du panel de l'enquête, bien qu'inscrits, afin de conserver l'homogénéité des données et d'avoir la possibilité de faire des comparaisons significatives.

La variable de la présence aux séminaires correspond à la mesure de l'assiduité aux réunions virtuelles synchrones obligatoires sur la plateforme avec le tuteur. Un premier groupe d'étudiants se distingue parce qu'ils se donnent les moyens de disposer de tout le temps nécessaire, soit que leurs institutions le leur ont accordé soit qu'ils le prennent au risque d'empiéter sur leurs obligations professionnelles et familiales. Ce groupe d'étudiants s'accroche contre vents et marées aux plannings proposés en début de cycle, et répond présent aux convocations aux réunions virtuelles dans $90 \%$ des cas. Cette caractéristique les fait définir comme "accrocheurs". Les deux autres groupes ont moins de temps disponible, soit qu'ils doivent s'acquitter d'obligations professionnelles incontournables, réduisant d'autant leur disponibilité, soit qu'ils ne ressentent pas l'obligation de venir sur la plateforme aux réunions virtuelles synchrones. Ils se différencient par le temps consacré aux réunions virtuelles synchrones avec leur tuteur : les "sérieux" sont présents dans $60 \%$ des séminaire virtuels tandis que les "indépendants" n’y viennent qu'à 30\% du temps total de connexion demandé.

La variable de la motivation de l'étudiant pour le travail d'équipe se mesure par l'observation de son implication dans les échanges virtuels, telle qu'elle transparaît dans les chats enregistrés au début des séminaires. Elle correspond à la participation aux négociations au sein de l'équipe, pour la définition des tâches, leur répartition, le calendrier de réalisation permettant d'établir le moment où chacun devra apporter le résultat de son travail personnel au groupe. Ce moment fort où l'équipe se soude ou n'arrive pas aux objectifs fixés, dure seulement quelques heures, cruciales pour le bon déroulement de la suite des opérations. Les "accrocheurs" montrent leur forte motivation en participant sous forme d'apport collaboratif à la presque totalité du temps d'échange consacré à la mise au point des activités à venir, tandis que les "sérieux" se contentent de consacrer $2 / 3$ de leur temps d'échanges à cette question tandis que les "indépendants" ne s'y intéressent que pour la moitié du temps déjà fort limité qu'ils consacrent aux échanges pendant cette période.

A partir de ces trois paramètres, il a été possible de quantifier le nombre d'étudiants entrant dans chacun des trois profils : les "accrocheurs" sont 26 (soit 28\% du total du panel), les "sérieux" 46 (soit 49\%) et les "indépendants" 22 (soit 23\%.). Le premier profil est perfectionniste par rapport au mode d'échanges collaboratifs en ce sens qu'il ne compte pas le temps passé dans les échanges et est par conséquent très exigeant en termes d'engagement réciproque de la part des autres membres de l'équipe. Le deuxième profil est relativement détaché du rythme imposé par la succession des séminaires à cause d'autres contraintes liées à leur réalité professionnelle. En effet, ce groupe a en général une pratique professionnelle non interrompue pendant l'année du DESS et par conséquent doit jongler entre les plages horaires prises par les obligations professionnelles et celles normalement consacrées au DESS. Le travail demandé par l'équipe est fourni souvent depuis le domicile, le soir et en week-end. Le troisième profil, sans réel engagement personnel pour collaborer, préfère travailler de manière indépendante. Ces personnes se contentent de témoigner de temps en temps de leur présence dans les séminaires lorsque la pression devient trop forte de la part du coordinateur qui est chargé de relancer les absents aux séminaires synchrones. Le résultat est plutôt décevant du point de vue du tuteur car elles n'arrivent pas à s'immiscer dans le processus de collaboration en ce sens qu'elles n'adhèrent pas aux protocoles d'échanges et d'élaboration d'une synthèse collective. 


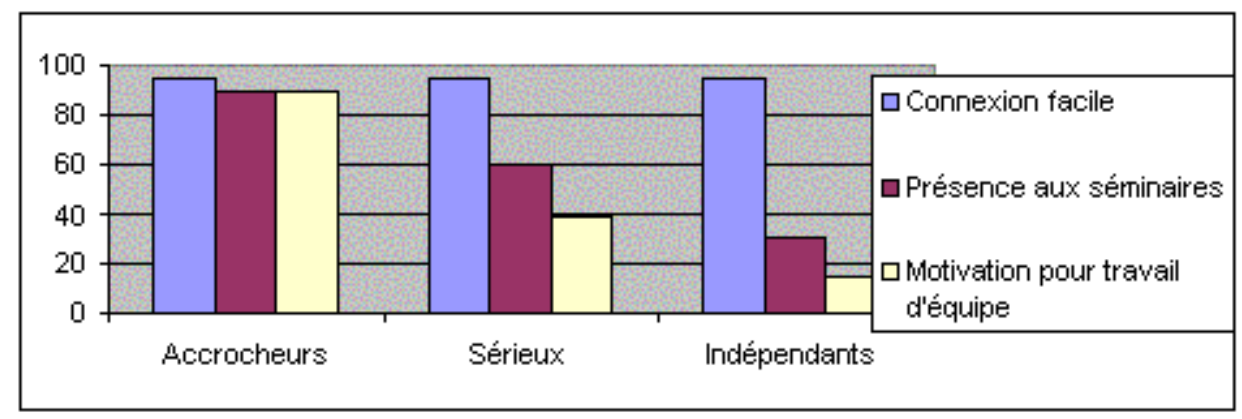

Tableau 1 : Profils des étudiants du DESS UTICEF

Les deux autres variables étudiées sont la participation aux réunions synchrones sans le tuteur et la collaboration à l'œuvre dans le travail d'équipe. La première tend à mesurer l'implication dans le travail d'équipe au travers des enregistrements de chats consultables après coup. Il s'agit de mesurer par rapport au total d'heures à envisager en réunions synchrones sans tuteur pour mener à bien le travail demandé sous forme collaborative, combien de temps l'étudiant a vraiment passé en réunion à cet effet. La deuxième variable tend à évaluer le degré de collaboration au travers de l'examen des activités de l'équipe, que ce soit les chats ou les versions successives des documents d'équipe déposés dans l'espace de travail correspondant. La collaboration correspond à une véritable mise en commun d'éléments rédigés par les équipiers, discutés et amendés avant d'être fondus dans la synthèse réalisée lors de la rédaction du document de travail final. Quand les étudiants sont forcés de travailler en groupe, ils peuvent se contenter d'apporter le résultat de leur travail à l'équipe de telle sorte que leur partie soit jointe telle quelle au document global, ce qui équivaut à une pratique de coopération et non de collaboration. L'appréciation du tuteur est utilisée pour évaluer le degré de collaboration de chaque membre de l'équipe par rapport à un modèle parfait qui consisterait à n'étudier que dans un contexte collaboratif. Le pourcentage retenu équivaut à la mesure du temps passé à travailler en collaboratif versus celui passé en solo ou en coopération.

On voit dans le graphe ci-dessous que la présence aux réunions synchrones avec le tuteur va de pair avec la participation active aux échanges sans le tuteur et au travail collaboratif d'équipe pour le profil des "accrocheurs" (à peu près $100 \%$ des cas). Dans ce cas, on peut souligner la corrélation entre la participation active aux réunions synchrones avec et sans tuteur et celle au travail de l'équipe, qui est la marque de fabrique en quelque sorte du modèle collaboratif du DESS UTICEF et qui justifie aux yeux de ses concepteurs, la coercition exercée sur les étudiants pour leur faire adopter ce mode de fonctionnement. Par contre, les "sérieux" sont moins souvent présents aux réunions sans tuteurs qu'aux réunions obligatoires avec tuteur ( $80 \%$ des cas), mais participent quand même aux échanges au sein de leur équipe pour collaborer ( $40 \%$ de leur temps d'étude). La question est de savoir quoi faire avec les étudiants réticents et jusqu'où il convient de ne pas aller trop loin en terme d'imposition de comportement d'apprentissage. Les "indépendants" sont souvent absents des réunions synchrones sans tuteur (20\%) et s'investissent beaucoup moins dans le modèle collaboratif proposé (10\% de leur temps d'étude). On peut constater que leur taux d'abandon et d'échec est plus important que celui des autres profils.

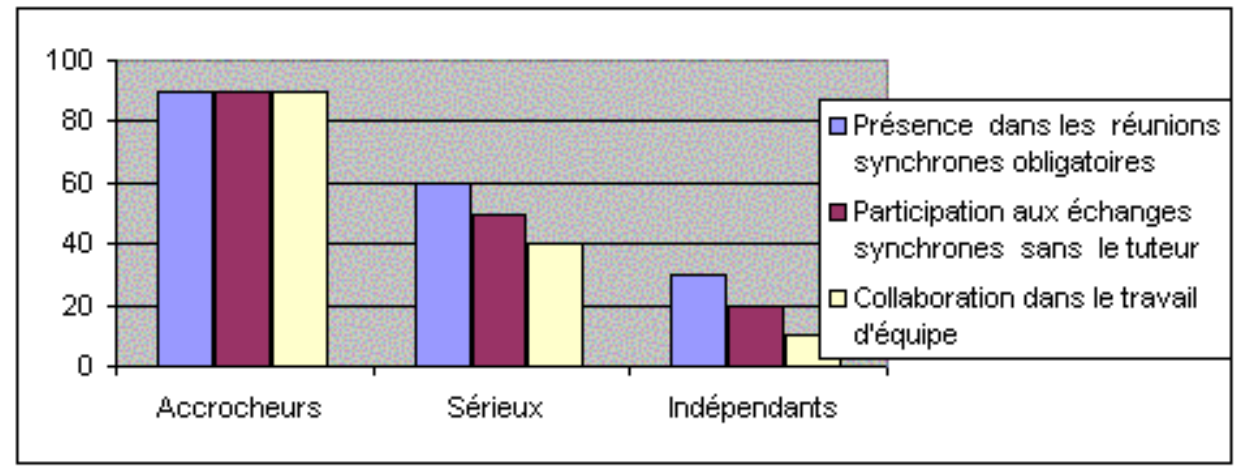

Tableau 2 : Différences de comportement des étudiants du DESS UTICEF

\section{Contraintes liées au modèle collaboratif}

Les premières études relatives au CSCL (Computer-Supported Collaborative Learning : apprentissage collaboratif assisté par ordinateur) ont été menées en France au CUEEP de Lille 1 depuis une dizaine d'années par Derycke et d'Halluin [DeryckeHalluin95]. Il a été constaté qu'il fallait forcer les étudiants à pratiquer la collaboration, qui ne leur était pas naturelle, $\mathrm{du}$ fait du poids des habitudes et des méthodes transmissives de leur passé. Chantal d'Halluin [Halluin01] a noté à propos des usages du campus virtuel de Lille 1 dans les points d'accès mis en place dans les villes et villages par les collectivités territoriales, que les étudiants n'utilisaient pas les forums et ne collaboraient pas naturellement. Comme le remarquent George et Leroux [GeorgeLeroux02], il est nécessaire de créer les conditions pour qu'il y ait une réelle activité collective entre apprenants 
afin de les inciter à interagir. Mais leur donner des outils techniques de communication est insuffisant pour les amener à interagir entre eux.

Les contraintes pédagogiques accompagnant l'apprentissage collaboratif ont pour objectif d'impliquer les étudiants dans le travail de groupe. Une situation de coercition est installée sciemment dès le début du DESS UTICEF : les étudiants sont invités à se plier au modèle proposé. La seule manière d'étudier proposée est de résoudre les situations problèmes en travaillant en collaboration avec ses équipiers, avec un système de notation en partie fondé sur la propension à collaborer. On observe en général des flottements et tergiversations durant les deux premiers mois avant que l'habitude de travailler en groupe ne soit vraiment prise ou bien complètement rejetée. Les compétences transversales à acquérir concernent des savoir faire relationnels à pratiquer sur les réseaux. Les problèmes viennent essentiellement d'engagements partiels qui ruinent la dynamique des équipes collaboratives. Très vite au cours des sessions, les étudiants identifient ceux qui s'investissent vraiment et ceux qui ne le font pas. Ces derniers sont peu appréciés car ils ne jouent pas le jeu; par conséquent, leur présence au sein d'une nouvelle équipe est contestée par les autres membres, si on leur en donne l'occasion. Non seulement la disponibilité est un facteur important mais aussi la capacité à produire les documents intermédiaires, permettant d'élaborer ensuite le document de synthèse de l'équipe. Le bouche-à-oreille fonctionne vite : certains étudiants se retrouvent isolés, faute d'avoir été acceptés par leurs partenaires, dans le cas de la composition libre des équipes. Toutes ces informations liées au comportement constaté, dessinent le profil de chaque étudiant et vont le définir par rapport aux autres de manière permanente au bout d'un certain temps de pratique collective.

\subsection{Limites du collaboratif par rapport à la taille des groupes tutorés}

Fjuk [FjukLudwigsen01] note à juste titre que les scénarios d'apprentissage collaboratif fonctionnent mieux avec de petits effectifs. La Téluq et l'Open University gèrent des groupes plus importants en développant une ingénierie spécifique. Dans le DESS UTICEF, une équipe composée de trois co-équipiers semble être la bonne taille pour les projets collaboratifs, où le troisième équipier est amené à jouer le rôle d'arbitre quand un désaccord se produit entre les deux premiers. Une équipe collaborative à quatre ou cinq équipiers peut également fonctionner à condition que personne ne cache son inaction derrière les autres. Les contraintes liées à la taille des groupes se combinent à celles relatives au rythme de travail demandé. Hotte [Hotte99] note que certains de ces groupes arrivent à former des collectifs intelligents d'apprentissage qui présentent un fonctionnement en mode autogéré reposant sur la présence de leaders de groupe.

En conséquence, la réaction du tuteur est attendue dans un délai le plus court possible, selon le principe de réciprocité : si l'équipe fournit des efforts importants, il est normal qu'elle obtienne un retour du tuteur le plus rapidement possible pour ne pas ralentir sa progression. Il s'agit en quelque sorte d'un contexte de préceptorat qui requiert un taux d'encadrement élevé et par conséquent cher. La pratique du DESS UTICEF fait que l'implication de l'encadrement en la personne du coordinateur, en plus des interventions personnalisées du tuteur, doit rester forte pour pouvoir relancer les étudiants dont la volonté de s'impliquer diminuerait. On peut considérer que le modèle du DESS UTICEF impose, outre un taux d'encadrement d'un tuteur pour 12/15 étudiants, un coordinateur pour une quarantaine d'étudiants. Autrement dit, appliquer un modèle collaboratif, implique un dispositif d'accompagnement conséquent à mettre en place.

\subsection{Limites du collaboratif par rapport à l'hétérogénéité des groupes}

Pour Reffay et Chanier [ReffayChanier02], s'appuyant sur les travaux d'Homans, l'estime réciproque pousse les étudiants à interagir de plus en plus entre eux. Ceci justement peut poser problème. Le prédicat est vrai dans le cas favorable d'étudiants motivés et de même niveau, il est infondé dans l'autre. On constate en effet avec le DESS UTICEF qu'une équipe collaborative trop hétérogène ne fonctionne pas. Pour créer une bonne dynamique, il vaut mieux mettre ensemble dans un groupe collaboratif en ligne, des étudiants de profils et de niveaux équivalents, avec des habitudes de travail similaires pour supprimer les effets de bord liés au rattrapage de certains par rapport à d'autres en termes de niveaux de connaissances et aussi aux blocages de certains étudiants par rapport à la pratique collaborative proposée. Où est l'intérêt du conflit socio-cognitif dans ce cas, si tout se passe entre pairs partageant les mêmes valeurs et objectifs, disposant à peu près du même bagage linguistique et des mêmes connaissances déclaratives ? La notion de niveau pour former les groupes est contraire semble-t-il à la philosophie de l'apprentissage collaboratif. Pourtant la recherche de l'homogénéité entre co-équipiers entraîne la création d'équipes différentes avec des niveaux différents. Par exemple, on pourra demander beaucoup plus en termes d'échanges collaboratifs à une équipe d'accrocheurs qu'à une autre composée d'indépendants. Scardamalia [Scardamania94] reconnaît que les meilleurs élèves tirent le mieux leur épingle du jeu collaboratif, comme dans tous les cas de figure d'ailleurs. Ils apportent une plus forte plus-value intellectuelle à la synthèse collective. Ils auront certainement tendance à travailler ensemble bien qu'ils soient recherchés par les autres. Quel est donc l'intérêt du collaboratif, s'il ne concerne d'abord et avant tout que les meilleurs ?

\subsection{Limites du collaboratif par rapport aux procédures d'évaluation}

Les modalités d'évaluation sont une source de difficultés du fait de la multiplicité des éléments à prendre en compte dans un cadre collaboratif, en particulier ceux relatifs aux échanges au sein du groupe de travail et à l'implication de chaque participant. L'apprentissage collaboratif repose sur le modèle socio-constructiviste tel que défini par Perret-Clermont [Perret74], avec une procédure d'évaluation plus complexe que dans le modèle transmissif car il s'agit d'évaluer non seulement les capacités d'assimilation et de synthèse de l'étudiant comme dans le modèle transmissif mais aussi sa propension à participer au travail d'équipe, à entraîner ses camarades et à mettre à leur disposition tout ce qu'il a accumulé dans le cadre de ses recherches personnelles. Mesurer l'apprentissage collaboratif est difficile : quelle proportion de la note est à consacrer à cet aspect, le reste étant affecté à l'évaluation du contenu (capacités de synthèse, d'expression écrite, etc.) ? La construction de la grille d'évaluation s'appuie sur la vérification de l'acquisition d'un savoir mais aussi sur l'observation des comportements et la qualité 
de la méthode de travail. La question non tranchée est celle de l'évaluation des activités collaboratives. On peut mesurer le degré d'implication de l'étudiant dans l'équipe de travail collaboratif. Mais cette question mérite d'être posée dans son intégralité : peut-on sanctionner les étudiants qui ont la malchance de faire partie d'une équipe qui n'a pas fonctionné en collaboratif ? Comment faire la part entre la bonne et la mauvaise volonté ? Il a été souvent constaté dans les trois promotions du DESS UTICEF que les équipes d'étudiants sérieux se contentent de se partager le travail de rédaction du document final sans collaborer, c'est-à-dire qu'elles optent pour la coopération, qui consiste à travailler chacun de manière isolée une fois que la répartition des tâches est effectuée. Dans ces conditions, si le document d'équipe rendu est de bonne qualité, faut-il baisser la note parce qu'il n'a pas été réalisé en collaboration?

Dans ce contexte, est-il justifiable de donner aux tuteurs les outils informatiques leur permettant de gérer plus d'équipes collaboratives en même temps ? George et Leroux [GeorgeLeroux02] s'appuient sur les travaux de Pléty du laboratoire d'éthologie des communications de Lyon 2 et sur sa typologie d'élèves répartis en modérateurs, évaluateurs, chercheurs et indépendants, les deux premiers étant très impliqués dans la collaboration, les deux autres beaucoup moins ou pas du tout. Ils transposent la typologie de Pléty sur les comportements des étudiants à distance avec l'objectif de donner un outil informatique au tuteur lui permettant de savoir à quel type appartient l'étudiant avec qui il interagit, basé sur le relevé de ses interactions dans les groupes de travail collaboratifs à distance. On peut en effet calculer le profil des étudiants en fonction du taux de participation aux discussions virtuelles des différentes équipes. De cette manière, le tuteur pourrait gérer les cas qui vont demander son interaction directe, les autres étant capables de se débrouiller entre eux. Reffay et Chanier [ReffayChanier02] proposent de bâtir des graphes qui reflètent les échanges au sein des groupes pour en mesurer la cohésion, basée sur les échanges entre membres d'un groupe par courrier électronique, forums et chats. Mais le premier résultat est qu'il n'est pas possible, pour le moment, d'identifier les sous-groupes les plus actifs vers lesquels pourtant la démarche était orientée afin que le tuteur puisse être informé de leurs performances. Il nous paraît hasardeux de développer de manière systématique des procédures d'évaluation reposant sur la participation aux équipes collaboratives. Outre le fait qu'il n'est pas prouvé qu'on apprend mieux de cette manière, il est exagéré de devoir forcer les étudiants à adopter un modèle dont les concepteurs sont persuadés de l'efficacité, sorte de manière d'imposition unidirectionnelle en complète contradiction avec l'essence du collaboratif qui est précisément l'engagement personnel et volontaire mais en aucun cas forcé.

\section{Conclusion}

Les comportements liés à l'enseignement présentiel, privilégiant le travail individuel, continuent à influencer certains étudiants à qui il est demandé de s'adapter aux pratiques de l'apprentissage collaboratif à distance mais qui préfèrent travailler seuls. Ce modèle est encore prégnant en France et dans les universités francophones du Sud. Il est possible que ce type de résistance s'estompe au fur et à mesure que l'habitude sera prise de travailler en groupe. Toutefois, cette donnée ne peut pas être facilement évacuée. De plus, d'autres questions se posent à propos des pratiques de l'apprentissage collaboratif, en particulier sur les procédures d'encadrement, de suivi et d'évaluation.

Les remarques précédentes nous poussent à adopter une attitude prudente quand il est question d'appliquer le modèle collaboratif à des cohortes d'étudiants plus importantes en contournant le problème du nombre maximum d'étudiants gérables en simultané par un tuteur. Les outils de suivi pédagogique permettent certes d'obtenir les profils d'étudiants à partir d'une analyse de leurs comportements dans les activités collaboratives. Mais si le calcul du profil de comportement est au point, quel en est l'usage pédagogique réel ? Il n'est peut-être pas souhaitable d'extrapoler à partir d'expériences collaboratives à petits effectifs pour en tirer des processus applicables grâce au développement d'outils informatiques, à de plus grands groupes.

Le tableau ne serait pas complet si nous ne mentionnions pas les communautés d'apprentissage fonctionnant sans tuteurs et dont il faudrait se demander si elles ne représenteraient pas une alternative à prendre en considération. Les communautés de pratiques sont largement utilisées dans ce contexte, comme l'a fait remarquer Wenger [Wenger01]. Les échanges se centrent sur une tâche professionnelle et concernent par exemple des médecins, des ingénieurs qui veulent parfaire leur savoir-faire sur un aspect bien particulier de leurs activités. Les experts du domaine étant les participants aux échanges, arrivent à synthétiser leurs savoirs en les échangeant avec d'autres. On peut penser que ce type de communauté peut fonctionner selon une dynamique propre, sans intervention systématique de l'enseignant.

Dans cette perspective, l'accent est à mettre plus sur les échanges de données et d'informations entre apprenants et enseignants que sur des comportements codifiés. La flexibilité et la réactivité deviennent les critères dominants pour mesurer la qualité de l'échange. Perriault [Perriault02] souligne l'importance de nouveaux modèles cognitifs d'apprentissage mobilisant l'induction, l'abduction, les capacité de travail en équipe, et de l'ouverture de la sphère de l'apprentissage en ligne vers des domaines tenus jusqu'à présent séparés concernant la recherche d'information et la gestion des connaissances. L'apprentissage en ligne est à la recherche d'un nouveau modèle qui rendrait compte de la diversité des usages présents et à venir. Ce nouveau modèle devra répondre à l'objectif de faciliter le plus d'échanges possible entre participants tout en privilégiant la simplification des procédures d'accès aux informations les plus pertinentes. 


\section{Références}

\section{Bibliographie}

\section{[Cerrato99]}

CERRATO T. (1999). Instrumenting collaborative writing and its cognitive tools. Human Centered Processes. $10^{\text {th }}$ Mini EURO Conference. Brest, France, (22-24 September), 141-143.

\section{[DeryckeHalluin95]}

DERYCKE A., \& D'HALLUIN C. (1995). Co-operative learning in the distance education of adults: why, how and first results from the CO-Learn project. Innovative Adult Learning with Innovative Technologies, Collis B. \& Davis G.(Eds), Elsevier Science B.V.

\section{[Halluin01]}

D'HALLUIN C. (2001). Usages d'un environnement médiatisé pour l'apprentissage coopératif. Les Cahiers d'Etudes du CUEEP, 43, 34-35.

\section{[FjukLudwigsen01]}

FJUK A. \& LUDWIGSEN S. (2001). The Complexity of Distributed Collaborative Learning: Unit of Analysis. Proceedings of EURO-CSCL'2001 Conference, Maastricht.

http://ww.mmi.unimaas.nl/euro-cscl/Papers/51.doc

\section{[GeorgeLeroux02]}

GEORGE S. \& LEROUX P. (2002). An Approach to Automatic Analysis of Learners' Social Behavior During ComputerMediated Synchronous Conversations. $6^{\text {th }}$ International Conference ITS 2002. Biarritz, France and San Sebastian, Spain, (June), Proceedings, Springer, 630-640.

\section{[GeorgeLeroux01]}

GEORGE S. \& LEROUX P. (2001). Un environnement support de projets collectifs entre apprenants : SPLACH. Sciences et Techniques Educatives, 8(1-2), 49-60.

\section{[Hotte99]}

HOTTE, R. (1999). L'EPAO : un environnement propice à la construction dynamique des connaissances. Sciences et Techniques Educatives, 6(2).

\section{[PeetersA199]}

PEETERS, R. , CHARLIER, B., DAELE, A., CHEFFERT, J.L., LUSALUSA, S. \& VIDA, T. (1999). Learning collaboratively in a virtual campus - Apprendre en collaborant dans un campus virtuel. Projet Learn-Nett : rapport du groupe de travail n ${ }^{\circ} 4$

\section{[Perret79]}

PERRET-CLERMONT, A.N. (1979). La construction de l'intelligence dans l'interaction sociale. Berne, Peter Lang.

\section{[Perriault02]}

PERRIAULT J. (2002). L'accès au savoir en ligne. Paris, Editions Odile Jacob.

\section{[ReffayChanier02]}

REFFAY C. \& CHANIER T. (2002). Social Network Analysis Used for Modelling Collaboration in Distance Learning Groups. $6^{\text {th }}$ International Conference ITS 2002. Biarritz, France and San Sebastian, Spain, (June), Proceedings, Springer, 31-41.

\section{[Scardamania94]}

SCARDAMALIA M. \& BEREITER C. (1994). Computer Support for Knowledge-Building Communities. The Journal of Learning Science, 3(3), 265-283.

\section{[TricotA198]}

TRICOT A., PIERRE-DEMARCY C. \& EL BOUSSARHINI R. (1998). Un panorama des recherches sur l'activité mentale de l'utilisateur d'un hypermédia, Sciences et Techniques Educatives, 5(4), 371-400. 


\section{[Wenger01]}

WENGER E. (2001). Supporting communities of practice : a survey of community-oriented technologies. http://www.ewenger.com/tech

\section{A propos des auteurs}

Michel Arnaud, maître de conférences à l'Université de Paris X Nanterre, a été responsable du DESS UTICEF (Utilisation des Technologies de l'Information et de la Communication pour l'Enseignement et la Formation) de l'Université Louis Pasteur, dispensé à distance auprès d'étudiants en France et dans les pays francophones du Sud, en partenariat avec le TECFA de l'Université de Genève, l'Université de Mons, l'Institut Supérieur de Documentation de Tunis et l'Agence Universitaire de la Francophonie. Il étudie les nouvelles configurations d'accès au savoir en ligne sous l'angle de la normalisation envisagée comme outil de démocratisation des usages, dans le cadre de l'AFNOR où il anime un groupe de travail sur l'apprentissage collaboratif et les logiciels ouverts.

Adresse : CRIS SERIES

Université Paris X Nanterre

200 avenue de la République

92001 Nanterre Cedex

Courriel : $\underline{\text { michel.arnaud@u-paris10.fr }}$

Référence de l'article :

Michel Arnaud, Les limites actuelles de l'apprentissage collaboratif en ligne, Revue STICEF, Volume 10, 2003, Rubrique, mis en ligne le 1511-2003, http://sticef.org, ISSN : 1764-7223 